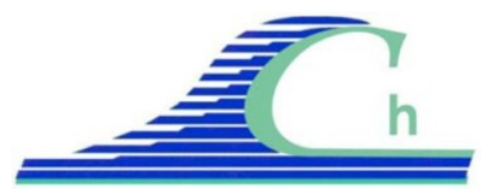

XII ${ }^{\text {èmes }}$ Journées Nationales Génie Côtier - Génie Civil

Cherbourg, 12-14 juin 2012

DOI:10.5150/jngcgc.2012.080-M C Editions Paralia CFL

disponible en ligne - http://www.paralia.fr - available online

\title{
Méthodes de protection contre la corrosion des ouvrages portuaires de génie civil
}

\author{
Faroudja MEZIANI ${ }^{1}$, Amar KAHIL ${ }^{1}$, Smail GABI $^{1}$, Aldjia BOUTIBA ${ }^{1}$
}

1. Université de Tizi-Ouzou, Faculté de génie de la construction,

Département de génie civil, BP 15000 Tizi-Ouzou, Algérie.mfaroudja@yahoo.fr ; amar_kahil@yahoo.fr;gsmail@yahoo.fr ; boualdjedi@yahoo.fr

\section{Résumé :}

Les parcs d'ouvrages portuaires comportent des structures complexes et variées. La connaissance de l'état de ces structures est une condition nécessaire à une bonne gestion. La démarche visant à combler le retard d'une maintenance préventive conduit à diagnostiquer une action prioritaire contre le processus de corrosion des aciers des structures métalliques et armatures des bétons. Les procédés ou techniques de prévention ou de protection de type électrochimiques sont détaillés par le biais de données émanant de cahiers des charges de chantiers à réaliser ou de récents chantiers réalisés en construction nouvelle ou en réhabilitation. Les méthodes sont comparées et les procédés de réhabilitation associés sont évoqués. Notre article se concentre sur la réparation de pieux corrodés en milieu marin.

Mots-clés :

Ouvrages portuaires - Corrosion - Protection - Milieux marins - Méthodes de protection

\section{Introduction}

Les ouvrages portuaires sont complexes en raison de la grande variété des types de structures et des modes de construction. L'agressivité liée au milieu maritime et à l'utilisation qui est faite des ouvrages induit des modes et des cinétiques de rupture et de dégradation spécifiques (CAUDE, 2008).

Le gestionnaire d'ouvrages portuaires est confronté à un double enjeu : assurer et maintenir un niveau de service convenable de tous ses ouvrages, compatible avec les critères économiques liés au fonctionnement du port et préserver la sécurité des personnes qui y ont accès. La connaissance la plus complète possible du parc est une condition nécessaire à l'application de toute méthode de gestion retenue et à son efficacité.

\section{Les ouvrages portuaires de génie civil}

Les ouvrages portuaires sont constitués de structures de complexité variable qui répondent à des contraintes de service spécifiques aux activités multiples d'un port, ainsi qu'à des contraintes environnementales sévères. Au cours du temps, en fonction des 
modifications d'exploitation le plus souvent, de nouvelles structures ont été superposées aux anciennes, celles-ci étant conservées en l'état ou détruites partiellement ou totalement suivant les critères d'exploitation retenus ou les niveaux de contraintes mécaniques adoptés.

Ainsi les ouvrages des parcs actuels présentent une diversité dans leurs typologies, dans les formes des structures et dans la nature des matériaux qui les composent. Dimensionnés pour répondre à de multiples sollicitations internes et extérieures, ils doivent répondre maintenant à des exigences de durabilité, particulièrement drastiques dans un environnement des plus agressifs relatif à l'ambiance "milieu marin" et au type d'exploitation des lieux (GODART et al., 2004).

\section{La maintenance}

Les gestionnaires d'infrastructures portuaires doivent maintenir le niveau de service des ouvrages pour respecter les exigences de leurs utilisateurs. Ils sont donc amenés à définir, programmer et réaliser des interventions d'entretien, de réparation ou de confortement d'ouvrages qui peuvent parfois être lourdes et coûteuses, surtout en cas des opérations purement curatives.

En matière de protection contre la corrosion de toute construction métallique, la simplicité et l'efficacité des solutions dépendent généralement du stade auquel elles sont élaborées.

Quelque soit la méthode utilisée, elle comporte les étapes suivantes: établissement de la liste exhaustive des ouvrages concernés, élaboration des dossiers d'ouvrages qui doivent comporter pour chaque ouvrage : l'identification, le relevé de son état lors de la dernière inspection (structure et matériaux), l'évolution des désordres observés, les causes de ces désordres et les préconisations de maintien à niveau, de remise à niveau ou de relèvement de niveau suivant les cas. Un classement des priorités « techniques » est doublé d'un classement de priorités "fonctionnelles", ce qui donne au gestionnaire la possibilité d'arrêter annuellement la liste des travaux prioritaires à effectuer avec le souci d'une gestion maitrisée dans le cadre du budget de maintenance alloué.

\section{Le processus de corrosion}

Par corrosion d'un métal, on entend la transformation du métal (élément fer) en divers composés (oxydes et hydroxydes) sous une action chimique (action de l'oxygène de l'air en présence d'eau) ou électrochimique (formation de micro-piles entre zones hétérogènes). On peut distinguer les facteurs de corrosion suivants :

a) Chimiques : oxygène, salinité, $\mathrm{pH}$, gaz carbonique.

b)Physiques : vitesse des courants, température, pression, particules solides présentes dans l'eau (sable, ...).

c) Biologiques : salissures marines biologiques végétales ou animales, bactéries.

Notre article se concentre sur la réparation de pieux corrodés en milieu marin. 


\section{XII ${ }^{\text {èmes }}$ Journées Nationales Génie Côtier - Génie Civil \\ Cherbourg, 12-14 juin 2012}

\section{Délimitation de la vulnérabilité de la corrosion marine}

La délimitation de la vulnérabilité de la corrosion marine peut être considérée d'une part, par le site (zones d'exposition au milieu marin et composition de l'électrolyte en contact), et d'autre part, par la cinétique de la corrosion de l'acier constitutif de la structure métallique d’un ouvrage maritime.

\subsection{Le site}

Le site abrité est généralement très différent du site ouvert. Dans un port, le risque de corrosion n'est pas le même lorsque le niveau de l'eau est constant (avec un faible marnage) ou lorsqu'il s'agit d'un milieu ouvert (avec un marnage élevé).

Il existe de nombreux ports à proximité ou à l'intérieur d'estuaires constituant un environnement très particulier : la salinité varie et il s'ensuit généralement un accroissement du taux de corrosion.

Les rejets industriels et l'élévation de température à proximité des centrales électriques sont également de nature à augmenter localement le taux de corrosion en site abrité.

\subsection{Le niveau}

La figure 1 représente uniquement la situation locale et il faut se garder de lui donner une signification plus générale. Néanmoins, elle représente des tendances qui, à trois niveaux spécifiques, sont particulièrement répandues, à savoir : zone des embruns, zone immergée et zone en fiche.

a) Zone des embruns : Cette zone possède un taux de corrosion très élevé, et cela pose le problème de la protection des ouvrages métalliques, avec la difficulté de maintenir un bon revêtement dans cette zone qui n’est pas immergée mais toujours humide.

b) Zone immergée : En immersion permanente, un peu au-dessous du niveau moyen de basse mer, il existe également un risque de corrosion assez marqué, dû à l'aération différentielle dont le processus est le suivant : à marée montante, l'eau vient recouvrir des parois fraîchement aérées et qui ont donc absorbé superficiellement une certaine teneur en oxygène. Il s'ensuit une tension d'électrode de cette paroi (aérée) plus positive (cathode) que celle de la paroi immergée (anode), et la différence de potentiel peut atteindre $0,1 \mathrm{~V}$, provoquant ainsi une véritable corrosion électrochimique de la zone non aérée.

c) Zone en fiche : En règle générale, les parties en fiche ou en contact avec le remblai ou les faces internes des pieux, ne subissent qu'une corrosion négligeable, car il se forme à la surface de l'acier un composé ferro-silicieux très dur qui bloque toute attaque ultérieure. De plus, l'action de l’oxygène est limitée dans le temps et en niveaux. 


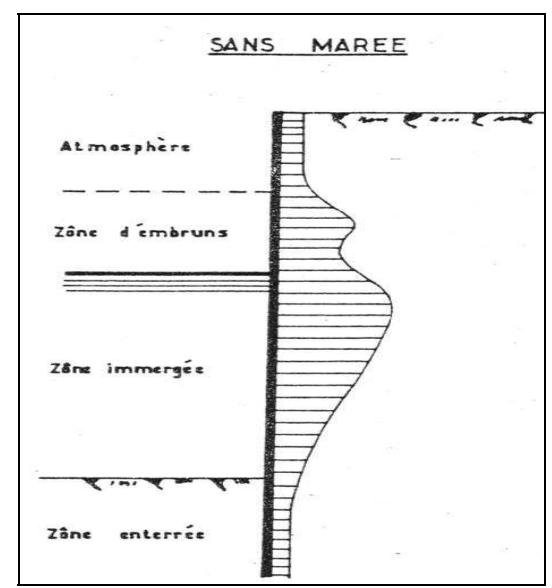

Figure 1. Vitesse de corrosion d'une structure immergée en milieu marin.

\section{Techniques de réparation des pathologies de corrosion des structures maritimes}

La délimitation de la corrosion marine peut être considérée d'une part, par le site, et d'autre part, par le niveau auquel s'exerce la corrosion sur un ouvrage maritime en acier. En milieu marin, la réussite de nombreuses applications illustre le bénéfice ainsi tiré d'une libre prédétermination, et confirme la nécessité d'analyser les risques de corrosion (cas d'une structure neuve) et les phénomènes de corrosion (cas d'une structure ancienne), (SCHOEFS \& REMOND, 2003).

\subsection{Les peintures anticorrosion}

Avant d'appliquer les différentes peintures, il faut que les pieux métalliques soient nettoyés. Ce nettoyage se fait en plusieurs étapes. Tout d'abord, 3 ouvriers font de l'hydrodécapage avec un nettoyeur haute pression (500 à 1000 bars max), qui permet d'enlever toutes les concrétions et une partie de la corrosion. Ensuite, il faut sabler la surface, qui permet de mettre le fer à "blanc" et de donner de la rugosité au pieu. Lorsque cette étape est réalisée, il faut aussitôt effectuer la peinture pour éviter que le pieu s'oxyde (apparition d'une couleur orange) et donc que la peinture n'ait plus aucune utilité (SCHOEFS et al., 2003).

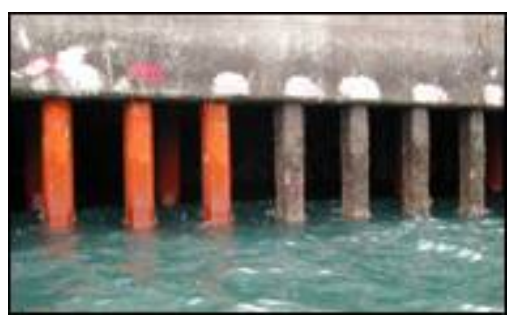

Figure 2. Pieux avant et après l'hydrodécapage.

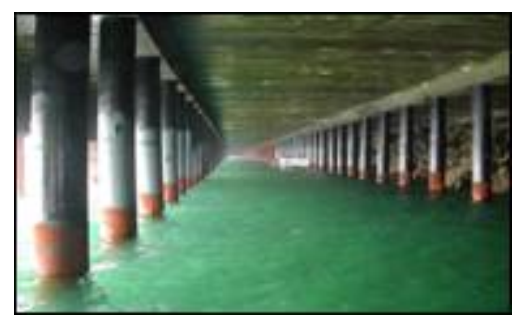

Figure 3. Essais des peintures sur pieux. 


\section{XII ${ }^{\text {èmes }}$ Journées Nationales Génie Côtier - Génie Civil \\ Cherbourg, 12-14 juin 2012}

\subsection{Réparation par recouvrement des pieux par le système Pile-Shield}

L'enveloppe Pile-Shield constitue une protection mécanique adaptée aux conditions sévères qui règnent en milieu marin. Il est combiné avec Pile Primer et Pile InnerWrap.

a) Pile Primer : Appliquer manuellement une fine couche de Pile Primer sur toute la surface en s'assurant que tout le métal exposé est protégé et donc enduire uniformément à la fois au-dessus et au-dessous de la ligne d'eau. Ensuite, il est nécessaire de frotter le Primer sur cette surface pour le faire pénétrer dans les 'pores' du métal en déplaçant l'humidité.

b) Pile Inner-Wrap : Commencer l'application par la partie basse de la surface à protéger. Les bandes à froid Pile Inner-Wrap seront enroulées en spirale avec un recouvrement minimum de 50\%. Tendre légèrement la bande, évacuer l'air en appliquant parfaitement la bande et favoriser ainsi un contact intime avec la canalisation.

c) Pile-Shield : Enrouler l'enveloppe autour du pilier en le plaquant contre les bandes Inner-Wrap et jusqu'à accrochage avec la fixation Velcro. Cercler avec une bande inox revêtue d'un isolant pour maintenir l'enveloppe en place. Appliquer la première bande inox au milieu de l'enveloppe et répartir les autres cerclages en respectant un espacement de 60 à $90 \mathrm{~cm}$. Les cerclages des extrémités seront positionnés à environ 30 cm du bord de l'enveloppe. Les enveloppes additionnelles seront positionnées à la suite et sans recouvrement.

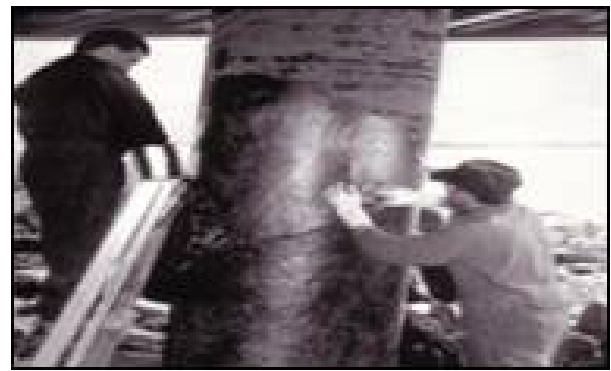

Figure 4. Mise en œuvre de l'enduit.

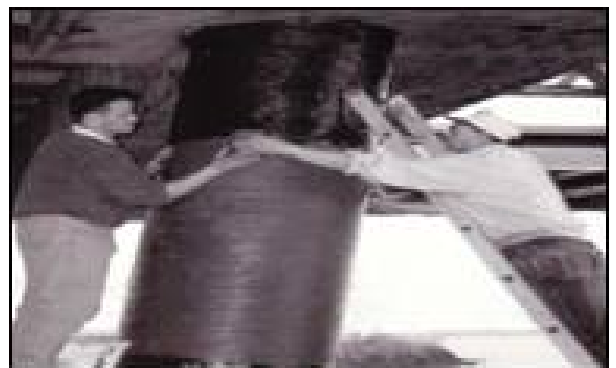

Figure 5. Mise en place des bandes à froid.

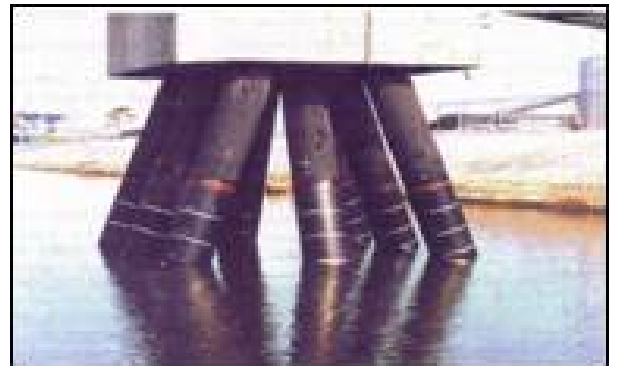

Figure 6. Pieux réparés. 


\subsection{Le système double coquille (méthode SOPROTEC)}

La marque SOPROTEC propose une méthode de protection de pieux contre la corrosion qui consiste à entourer le pieu d'une double coquille (SCHOEFS et al., 2003).

Tout d'abord, chaque demi-coque est recouverte d'une résine sur toute sa surface interne. Cette étape est réalisée juste avant la mise en place. Ensuite, on vient positionner une paire de demi-coque au niveau d'un point de rouille. Grâce à un système de vis, on va serrer et rapprocher les deux demi-coques sur le pieu. Ceci entraîne un refus de la résine ce qui va assurer une étanchéité parfaite entre le pieu corrodé et les deux demi coques.

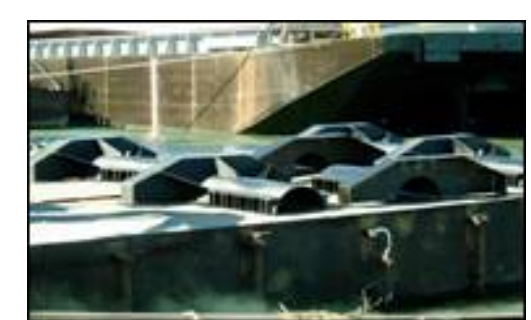

Figure 7. Exemple de demi-coquille.

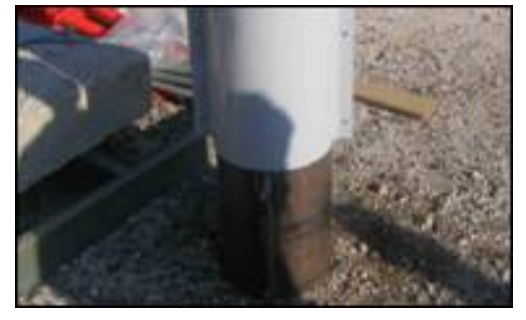

Figure 8. Coquille boulonnée.

\subsection{La protection cathodique}

Il existe deux méthodes pour fournir les électrons servant à polariser la surface :

a) Les systèmes par anodes sacrificielles: dans lesquels le courant de protection provient d'un métal dont le potentiel de corrosion est plus électronégatif que celui de la pièce à protéger (alliage d'aluminium, de zinc ou de magnésium pour l'acier) (PAUGAM et al., 2011).

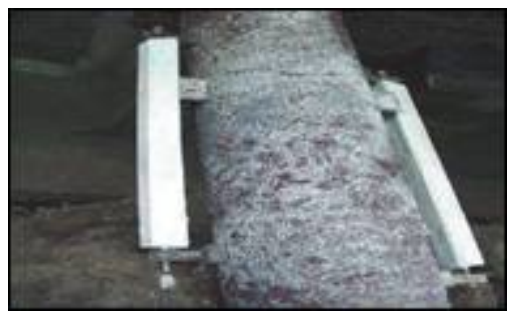

Figure 9. Anodes fixées sur pieu.

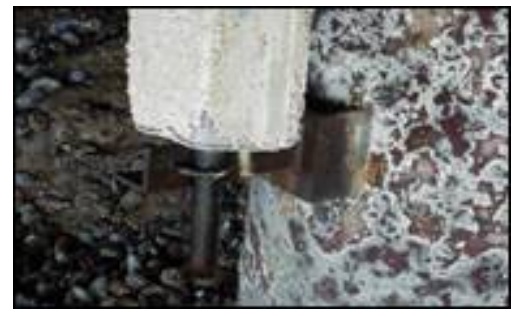

Figure 10. Dispositif de fixation.

b) Les systèmes par courant imposé où le courant continu : utilisé en association avec des anodes relativement inertes comme le graphite. Une installation électrique doit être prévue. Plusieurs dizaines de kilomètres de câbles électriques sont ainsi tirés des appontements au générateur.

Le tableau 1 indique la comparaison entre un système par anodes galvaniques et un système à courant imposé. 


\section{XII ${ }^{\text {èmes }}$ Journées Nationales Génie Côtier - Génie Civil \\ Cherbourg, 12-14 juin 2012}

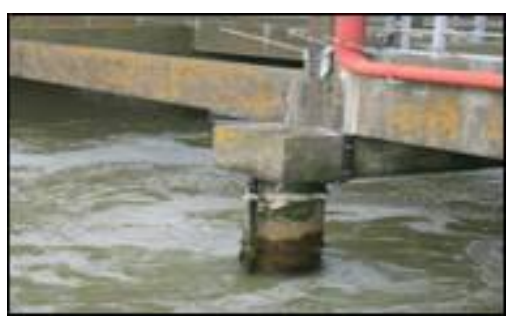

Figure 11. Câble gainé.

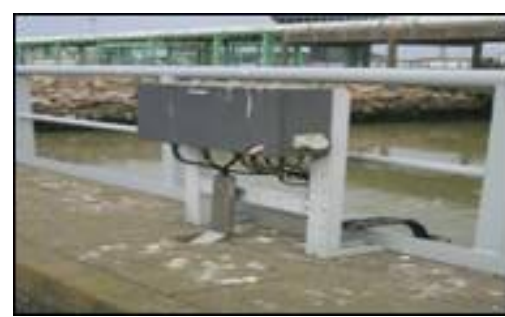

Figure 12. Générateur de tension.

Tableau 1. Comparaison des deux méthodes.

\begin{tabular}{|c|c|c|}
\hline & Systèmes par anodes galvaniques & Systèmes à courant imposé \\
\hline Environnement & $\begin{array}{l}\text { Utilisation pouvant s'avérer parfois } \\
\text { impossible sauf dans des sols ou les } \\
\text { eaux à faible résistivité. }\end{array}$ & $\begin{array}{l}\text { Utilisation moins dépendante de la } \\
\text { résistivité du sol ou de l'eau mais } \\
\text { pouvant générer } \mathrm{Cl}_{2} \text { en eau de mer. }\end{array}$ \\
\hline Installation & Facile. & $\begin{array}{l}\text { Nécessité d'une étude soignée pour } \\
\text { éviter les complications. }\end{array}$ \\
\hline Source d'énergie & $\begin{array}{l}\text { Indépendant de toute source } \\
\text { d'énergie. Il ne peut y avoir d'erreur } \\
\text { de branchement. }\end{array}$ & $\begin{array}{l}\text { Source extérieure indispensable. } \\
\text { Nécessité de faire attention aux erreurs } \\
\text { de branchement. }\end{array}$ \\
\hline Anodes & $\begin{array}{l}\text { Le volume des anodes galvaniques } \\
\text { peut perturber l'écoulement de l'eau, } \\
\text { créer des turbulences et des effets de } \\
\text { traînée. }\end{array}$ & $\begin{array}{l}\text { Les anodes inertes sont en général, plus } \\
\text { légères et moins nombreuses. Leur } \\
\text { forme peut être étudiée pour provoquer } \\
\text { une gêne minimale sur l'écoulement de } \\
\text { l'eau. }\end{array}$ \\
\hline Entretien & $\begin{array}{l}\text { En général, pas nécessaire. Le } \\
\text { remplacement des anodes est possible } \\
\text { dans certains cas. }\end{array}$ & $\begin{array}{l}\text { Matériel conçu pour une longue durée } \\
\text { de vie, à condition d'effectuer des } \\
\text { contrôles réguliers du matériel } \\
\text { électrique en service. }\end{array}$ \\
\hline Avaries & $\begin{array}{l}\text { Les anodes sont robustes et peu } \\
\text { susceptibles de subir des avaries } \\
\text { mécaniques. }\end{array}$ & $\begin{array}{l}\text { Les anodes inertes sont de construction } \\
\text { plus légère donc moins résistantes aux } \\
\text { avaries mécaniques. }\end{array}$ \\
\hline
\end{tabular}

\subsection{Protection des zones de liaison poteaux et sous faces béton}

Avant de mettre en place le mortier il faut réaliser un nettoyage de la zone à traiter pour enlever la laitance du béton, les concrétions, les résidus de mastic de coffrage et le revêtement de protection bitumineux ; afin d'avoir un support propre et donc une bonne adhérence. Le nettoyage dépend de la vétusté du pieu et de son degré de détérioration. En effet, un brossage métallique est amplement suffisant pour les pieux assez récents, mais à l'opposé, les plus anciens nécessitent obligatoirement un sablage.

Après avoir nettoyé le support, il faut préparer le mortier (constitué de deux composants, résine et durcisseur). Ces derniers doivent être soigneusement mélangés au 
moment de l'emploi sous agitation mécanique (200 à 300 tours par minute) jusqu'à l'obtention d'une pâte uniformément grise exempte de filaments. La mise en œuvre du produit est ensuite réalisée (assez aisément) à l'aide d'une spatule crantée ou d'une truelle.

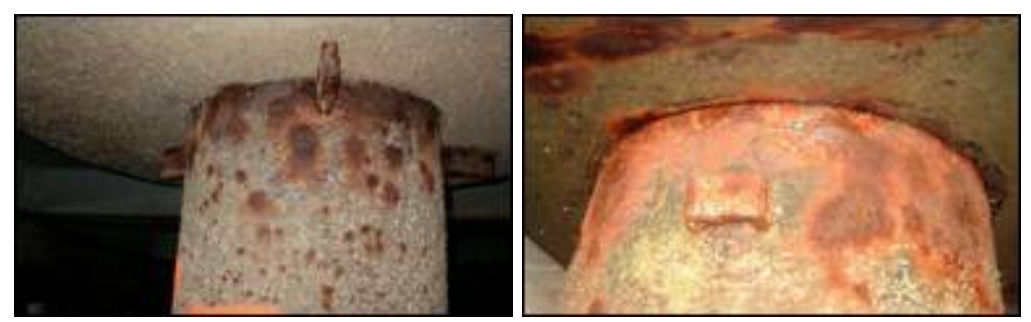

Figure 13. Exemples de corrosion sur la tête de pieu.

\section{Conclusion}

Les ouvrages en milieu marin sont exposés à des agressions importantes, essentiellement de la part des chlorures qui accélèrent le processus de corrosion des aciers. De nombreux procédés sont connus pour réparer des pieux (peinture, anode sacrificielle, ...) mais il est sans cesse tenté de les améliorer. C’est pourquoi de nombreux tests expérimentaux sont effectués. Ils permettront, par la suite de trouver les réparations les plus adaptées.

\section{Références bibliographiques}

CAUDE G. (2008). Pathologies des ouvrages portuaires : méthodes d'investigation. France, $90 \mathrm{p}$.

GODART C., DAGBERT C., GALLAND J. (2004). Impact de l'environnement marin sur la formation du dépôt calco-magnésien: rôle de la matière organique. Matériaux et Techniques 8, pp 27-32. doi:10.1051/mattech:2004033

PAUGAM L., FESTY D., PINEAU S., MENARD N., CARPENTIER P., BENAÏSSA B. (2011). Comportement électrochimique des structures métalliques portuaires en site marnant maritime et estuarien : monitoring de critères de protection cathodique. $5^{\text {èmes }}$ journées d'Aix de la Protection Cathodique - Théorie et pratique pour tous secteurs d'application, pp 1-16.

SCHOEFS F., REMOND F. (2003). Etude de risque appliquée à un quai en gabions soumis à de la corrosion, Rapport, Institut de Recherches en Génie Civil et Mécanique, $89 \mathrm{p}$.

SCHOEFS F., ANDRE M., BAZIN J., FOUACHE C., PAYRAUDEAU H. (2003). Techniques de réparation des pathologies de corrosion des structures métalliques analyse technico- économique. Rapport, France, 34 p. 